\title{
Phronesis
}

\section{Interagir pour apprendre en gestion de classe au secondaire : analyse du discours des futurs enseignants dans un espace collaboratif \\ Learning-as-participation in a collaborative environment for high school preservice teachers classroom management, a discours analysis}

\author{
Pier-Ann Boutin, Christine Hamel et Josée-Anne Gouin
}

Volume 5, numéro 3-4, 2016

Quels discours pour quel développement professionnel ?

URI : https://id.erudit.org/iderudit/1039088ar

DOI : https://doi.org/10.7202/1039088ar

Aller au sommaire du numéro

Éditeur(s)

Université de Sherbrooke

Champ social éditions

ISSN

1925-4873 (numérique)

Découvrir la revue

Citer cet article

Boutin, P.-A., Hamel, C. \& Gouin, J.-A. (2016). Interagir pour apprendre en gestion de classe au secondaire : analyse du discours des futurs enseignants dans un espace collaboratif. Phronesis, 5(3-4), 87-99.

https://doi.org/10.7202/1039088ar

\section{Résumé de l'article}

Afin de soutenir le développement professionnel des futurs enseignants au secondaire lors de leur formation initiale universitaire, et ce, en lien avec le concept de praticien réflexif dans une approche par compétence, un design pédagogique novateur fut mis en place dans un cours dédié à la gestion de classe. En effet, ce design, inspiré des principes de la communauté d'apprentissage (Brown, 1994), a amené les étudiants-stagiaires à développer un discours collectif sur leur pratique professionnelle à travers les enjeux propre à la gestion de classe au secondaire et à leurs préoccupations lors d'un stage de cinq semaines. Nous faisons la prémisse que la participation à l'élaboration d'un discours collectif sur les pratiques professionnelles permet aux étudiants de, non seulement, réfléchir sur leur pratique, mais de faire des apprentissages professionnels durables. Ainsi, notre étude s'attarde à l'engagement des étudiants dans l'élaboration du discours collectif et aux actions leur permettant de faire avancer celui-ci. 


\title{
Interagir pour apprendre en gestion de classe au secondaire : analyse du discours des futurs enseignants dans un espace collaboratif
}

\author{
Pier-Ann BOUTIN, Christine HAMEL et Josée-Anne GOUIN
}

\author{
Département d'études sur l'enseignement et l'apprentissage \\ Université Laval \\ 2320 rue des Bibliothèques, bureau 1238 \\ Québec (Québec, Canada) G1V OA6 \\ pier-ann.boutin@fse.ulaval.ca \\ christine.hamel@fse.ulaval.ca \\ josee-anne.gouin@fse.ulaval.ca
}

Mots clés : Discours collaboratif, futurs enseignants, apprentissage, interaction

Résumé : Afin de soutenir le développement professionnel des futurs enseignants au secondaire lors de leur formation initiale universitaire, et ce, en lien avec le concept de praticien réflexif dans une approche par compétence, un design pédagogique novateur fut mis en place dans un cours dédié à la gestion de classe. En effet, ce design, inspiré des principes de la communauté d'apprentissage (Brown, 1994), a amené les étudiants-stagiaires à développer un discours collectif sur leur pratique professionnelle à travers les enjeux propre à la gestion de classe au secondaire et à leurs préoccupations lors d'un stage de cinq semaines. Nous faisons la prémisse que la participation à l'élaboration d'un discours collectif sur les pratiques professionnelles permet aux étudiants de, non seulement, réfléchir sur leur pratique, mais de faire des apprentissages professionnels durables. Ainsi, notre étude s'attarde à l'engagement des étudiants dans l'élaboration du discours collectif et aux actions leur permettant de faire avancer celui-ci.

\section{Title : Learning-as-participation in a collaborative environment for high school preservice teachers class- room management, a discours analysis}

Keywords : collaborative environment, learning, interaction, preservice teachers

Abstract : In order to support the professional development of high school preservice teachers during their initial university training as reflective practitioners an innovative educational design was placed in a classroom management course. With this design, inspired by the principles of the learning community (Brown, 1994), has assisted students to develop a collective discourse on their professional practice through their own issues in the secondary classroom management and their concerns during a five weeks internship. We make the assumption that the participation in the development of a collective discourse on professional practice allows students to not only reflect on their practice, but also to gain significant professional learning. Thus, our study focuses on student engagement in the development of collective discourse and actions enabling them to advance it. 


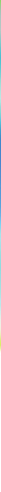

\section{Introduction}

\section{Définir un espace pour la construction d'un discours professionnel}

L'approche par compétences et le processus de professionnalisation mis de l'avant dans la plupart des programmes de formation à l'enseignement au Québec nécessitent de développer la réflexion sur la pratique chez les futurs enseignants. Cette réflexion sur la pratique leur permet de comprendre comment ils agissent, de s'interroger sur les choix et les effets de leurs actions. Elle leur permet également d'être en mesure de réinvestir leurs réflexions dans une nouvelle situation professionnelle (Korthagen, Loughram, \& Russel, 2006). Schön (1983) parle d'une réflexion dans l'action (pendant) et sur l'action (après). C'està-dire que le futur enseignant en stage va au-delà de la justification ou l'autosatisfaction pour expliquer ses actions et mise sur l'objectivation du processus qu'il a mis en place dans une situation professionnelle.

La réflexion sur la pratique entraine certains défis. Perrenoud (2001) posait d'ailleurs une question cruciale : « Comment développer une posture et des compétences proprement réflexives en formation des enseignants ? ». Pour lui, saupoudrer les cours universitaires de quelques séminaires à caractère réflexif est nettement insuffisant. La réflexion sur la pratique n'est pas un savoir en soi, mais un rapport au savoir, une posture critique dont le développement devrait être omniprésent dans toute la formation universitaire afin de développer la professionnalisation des futurs enseignants. Pour Tardif (2012), la professionnalisation constitue un processus de transformation d'une personne novice en un professionnel reconnu. Wittorski (2001) apporte une distinction entre formation professionnelle et formation professionnalisante. La formation professionnalisante forme aux outils, et aux méthodes de la profession comme dans la formation professionnelle, mais dans une perspective de réflexion directement liée aux pratiques préconisées par le groupe professionnel auquel l'apprenant veut appartenir. L'enjeu de la formation professionnalisante est donc la construction de l'identité professionnelle en lien avec son propre rapport à la profession et aussi, en confrontant l'identité collective de cette dernière. Le concept de professionnalisation renvoie au développement professionnel du futur enseignant afin qu'il devienne un praticien réflexif. Peu de temps et d'espace seraient consacrés à la réflexion sur la pratique dans le parcours de formation initiale en enseignement (Tardif et Desbiens, 2014). De fait, il existe peu d'espace pour la construction d'un discours professionnel durant la formation. Pour Rabatel et Blanc (2011), les discours professionnels sont des espaces de réflexion essentiels qui sont des lieux de négociations de sens et de construction de connaissances où se s'élabore une réflexion sur la pratique.

Afin de soutenir la construction d'un discours professionnel, nous avons élaboré un design pédagogique alliant discussions synchrones et asynchrones supportées par des rencontres face-à-face et un forum de coélaboration de connaissances (Knowledge Forum). Le modèle de la communauté d'apprentissage (Brown, 1997) nous a semblé le plus prometteur pour soutenir des interactions engageantes entre les étudiants de même que le développement d'un discours collectif porteur de sens dans le but d'approfondir leur compréhension et de résoudre des problèmes authentiques de gestion de classe. Comment le design pédagogique du cours, par l'espace fourni, peut-il soutenir la professionnalisation du futur enseignant autour d'une problématique forte qui concerne le métier? Dans cet espace, nous nous sommes intéressées aux interactions à l'écrit entre étudiants sur les enjeux de la gestion de classe au secondaire, une thématique importante qui comporte une forte part de craintes chez les futurs enseignants du secondaire au Québec (Hamel, Laferrière \& Allaire, 2012).

\section{Interagir en collaboration pour apprendre un métier entre débutants}

L'étudiant apprend en se basant sur ses perceptions initiales, intuitives ainsi que sur ses connaissances antérieures sur un sujet, un phénomène ou un concept tout en se concentrant davantage sur la construction de connaissances (Piaget, 1977). La perspective socioconstructiviste, quant à elle, insiste sur l'élément social qui mène à l'apprentissage, soit l'élève qui apprend en interaction avec les autres et avec son environnement. C'est sur ces apports fondamentaux de Piaget et de Vygotski que se basent plusieurs chercheurs 
en sciences de l'apprentissage (voir entre autres, Bereiter \& Scardamalia, 2010; Bransford, Brown, \& Cocking, 2000; Bruner, 1967; Perkins, 1995; Scardamalia \& Bereiter, 2003). L'interaction avec ses pairs donne ainsi à l'apprenant l'occasion d'exprimer et de confronter ses idées, de les remettre en question et surtout de négocier avec les autres en vue d'avoir une compréhension commune et viable du problème en cause. C'est dire que l'interaction en salle de classe peut s'avérer très riche lorsque les échanges ou les partages entre apprenants et avec l'enseignant permettent à l'étudiant de se construire une représentation « nouvelle », une connaissance plus approfondie d'un sujet donné.

Pour y parvenir, il est important pour l'enseignant de soutenir l'apprentissage des étudiants et le concept d'étayage (Scaffolding) fait explicitement référence au concept de zone de développement proximal ${ }^{1}$ en introduisant un individu plus expérimenté dans le processus afin de faciliter la résolution de problème. Traditionnellement, l'individu plus expérimenté dans la classe était l'enseignant. Cependant, l'étayage n'est pas réservé à l'interaction entre un enseignant et un élève, mais peut également s'appliquer lors d'interaction entre des élèves (Lai \& Law, 2006). L'étayage est en fait l'interaction d'un enseignant ou d'un pair, ayant une plus grande expérience avec un pair « moins » expert afin qu'il progresse (Mugny \& Doise, 1978). Les étudiants progressant dans une classe communauté d'apprenants et amorçant un processus de coélaboration de connaissances négocient et partagent afin de faire avancer leurs connaissances collectives sur un sujet (Brown \& Campione, 1996).

\subsection{Interaction et collaboration dans l'élaboration d'un discours collectif}

Dans le cas qui nous occupe, les étudiants ont à négocier leur compréhension de la gestion de classe à la lumière d'une pratique professionnelle émergente. Pour Piaget (1928), l'interaction entre pairs qui amène différentes idées et perspectives sur un sujet peut créer un conflit cognitif susceptible d'entrainer la construction d'une nouvelle structure conceptuelle, un apprentissage. De surcroit, selon Brown (1994), la négociation de sens qui a lieu entre les participants d'une communauté d'apprenants afin de discuter de leur conception d'un problème ou d'un concept favorise l'apprentissage de l'ensemble de la communauté :

Group cooperation, where everyone is trying to arrive at consensus concerning meaning, relevance, and importance, helps ensure that understanding occurs, even if some members of the group are not yet capable of full participation. Because thinking is externalized in the form of discussion, beginners can learn from the contributions of those more expert than they. (Brown, 1994, p.7)

Afin d'entrer dans une communauté, il nous faut en connaitre le langage, y participer de façon graduelle, périphérique, pour ensuite nous y intégrer en tant que membre (Sfard, 1998). Dans notre contexte, les étudiants en formation initiale à l'enseignement sont invités à participer activement aux séances en classe et à l'élaboration du discours collectif de la communauté sur support numérique. La communauté d'apprentissage met l'accent sur la métaphore de la participation pour apprendre (Sfard, 1998). Selon Sfard, la métaphore de la participation conçoit l'apprentissage comme un processus qui inclut une variété de pratiques culturelles et des activités de partage. L'accent est davantage sur le processus « apprendre » que sur le produit « connaissances » (Paavola, Lipponen et Hakkarainen, 2004). Cette façon de comprendre l'apprentissage met la communauté et la participation de ses membres au centre du processus par lequel on apprend. Entrer dans une pratique professionnelle c'est s'approprier, par l'entremise de la négociation de sens, un ensemble de valeurs, de connaissances et de schèmes qui forgeront l'identité professionnelle de l'enseignant en devenir. Cette identité professionnelle sera, non seulement teintée par les valeurs et connaissances reconnues dans le monde de l'enseignement au Québec, mais aussi par la culture de la commission scolaire, de l'école qu'ils fréquenteront, les expériences qu'ils vivront en tant que jeunes enseignants et par les interactions avec leurs pairs expérimentés ou non. Cette identité, cette façon d'appréhender l'enseignement et l'apprentissage se développent tout au long de la formation initiale où les stages sont un moment privilégié. Nous voulions donc en arriver à trouver un espace dans lequel les étudiants construiraient un discours professionnel pour confronter des idées, des savoirs et consigner leurs savoirs professionnels (Rabatel et Blanc, 2011).

Dans notre contexte universitaire, il nous a fallu développer un design pour faciliter la mise en œuvre des ces concepts pédagogiques forts puisque nous avions affaire à des sections nombreuses d'étudiants avec très peu de cours en classe. En effet, les deux cours de gestion de classe offerts aux étudiants avant et après leur troisième stage de cinq semaines constitue un moment pivot où ils sont amenés à interagir avec leurs pairs, non seulement durant les séances en classe, mais aussi sur un forum électronique, le Knowledge Forum (KF). Le but de ces interactions est l'élaboration d'un discours collectif professionnel par et pour les étudiants afin d'approfondir leurs connaissances sur la gestion de classe en lien avec le développement de leur identité professionnel.

Or, l'élaboration d'un discours collectif écrit ne va pas de soi, même en contexte formel d'apprentissage. L'élaboration d'un discours collectif écrit s'ancre au principe d'écriture transformative (Allaire et al., 2014; Bereiter et Scardamalia, 1987) qui vise l'appropriation de connaissances. Ainsi, l'acte d'écrire pourrait amener le participant à avoir de nouvelles idées, à faire de nouveaux liens entre des

1 La zone de développement proximale (Zones of Proximal Development, Vygotsky, 1978) est considérée comme la distance entre le niveau de développement actuel de l'apprenant et le niveau de développement qu'il peut atteindre en étant assisté par un pair ou une personne plus expérimentée. 
concepts. Ce processus pourrait être encore plus dynamique et fécond lorsque l'écriture est collective (Bereiter et Scardamalia, 1987). Cette façon d'appréhender l'écriture est nouvelle pour les étudiants qui la perçoivent plus souvent comme une activité individuelle, un processus de récitation de connaissances (knowledge telling, Allaire et al., 2011, 2013, 2014; Bereiter et Scardamalia, 1987) où ils colligent ce qu'ils pensent, ce qu'ils ont appris sur un sujet.

Les idées et les savoirs des étudiants sont non seulement confrontés à ceux de leurs pairs, mais aussi remis en question par leur propre expérience de stage. C'est donc un moment propice à la négociation de sens, qui nous apparait plus fécond encore si l'on y ajoute un aspect de collaboration. Le fait de faciliter et soutenir les interactions dans un environnement numérique asynchrone peut favoriser la collaboration entre les participants (Murphy, 2004). La collaboration est alors vue comme un processus de création partagée (Scharge, 1995), dont l'intention est de produire quelque chose pour résoudre un problème, de créer des artéfacts (Murphy, 2004 ; Schrage, 1995) et de travailler ensemble pour atteindre des objectifs communs (Kaye, 1992; Roschelle et Teasley, 1995). En étant exposés au point de vue des uns et des autres, les participants commencent à en tenir compte, à les négocier, à se les approprier (Murphy, 2004 ; Henri, 1995; Knuth et Cunningham, 1993; Jonassen et al, 1993). Cette étape est un préalable à la construction des connaissances et la construction de nouvelles significations (Murphy, 2004 ; Garrison et al, 2000; Schrage, 1995; Alexander, 1992; Henri, 1995). Ainsi, pour que la collaboration survienne lors de l'élaboration d'un discours collectif, il faut passer par différents niveaux d'engagement dans ce discours. Ainsi, le premier niveau est la participation au discours, c'est-à-dire la lecture des contributions des pairs et la contribution individuelle. Par exemple, développer une idée ou une opinion dans une contribution qui ne serait pas liée aux contributions des pairs par des liens d'enfilade (affordance du KF) et qui ne serait pas suivie par une élaboration de la part d'un autre étudiant. Le second niveau d'engagement est l'interaction, ici nous entendons par interaction les contributions en réponse à une autre, une élaboration qui n'est pas suivie par d'autres contributions. Au troisième niveau d'engagement se trouve la collaboration, elle englobe toutes les contributions ayant une suite de plusieurs élaborations (Zhao, Sullivan, \& Mellenius, 2013).

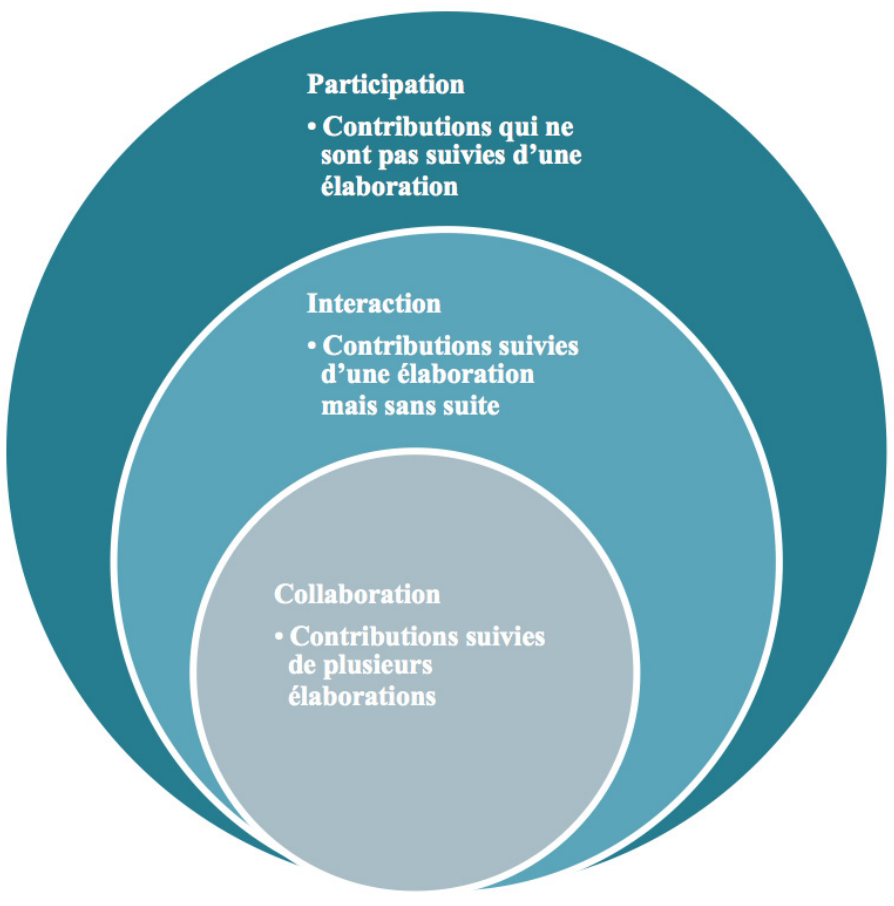

Figure 1 : Niveaux d'engagement dans le discours collectif (Zhao, Sullivan, \& Mellenius, 2013)

Afin de préciser les processus d'interaction entre les étudiants lors de l'élaboration du discours collectif, nous nous sommes basées sur les travaux de Henri $(1992,1995)$. L'auteur y propose une méthode d'analyse de contenu fondée sur types d'interaction entre les participants dans le cadre d'échanges écrits survenus lors de conférences virtuelles. Les types d'interactions réfèrent à l'intention d'écriture des participants et à l'interaction au sens large entre ceux-ci. En effet, Henri (1992, 1995) identifie trois types d'interactions : les interaction explicites, les interactions implicites et les interactions indépendantes. II importe toutefois de décrire plus qualitativement le contenu des contributions composant le discours collectif afin que la seule inte- 
raction progressive ne soit pas notre barème de compréhension de l'espace collectif en construction. Les travaux de Bielaczyc et Ow (2013) portent sur les actions posées lors de la résolution de problèmes complexes en collaboration. Les auteurs y distinguent différentes actions de base de l'investigation collective dans le processus de construction de connaissances comme le montre la figure 2.

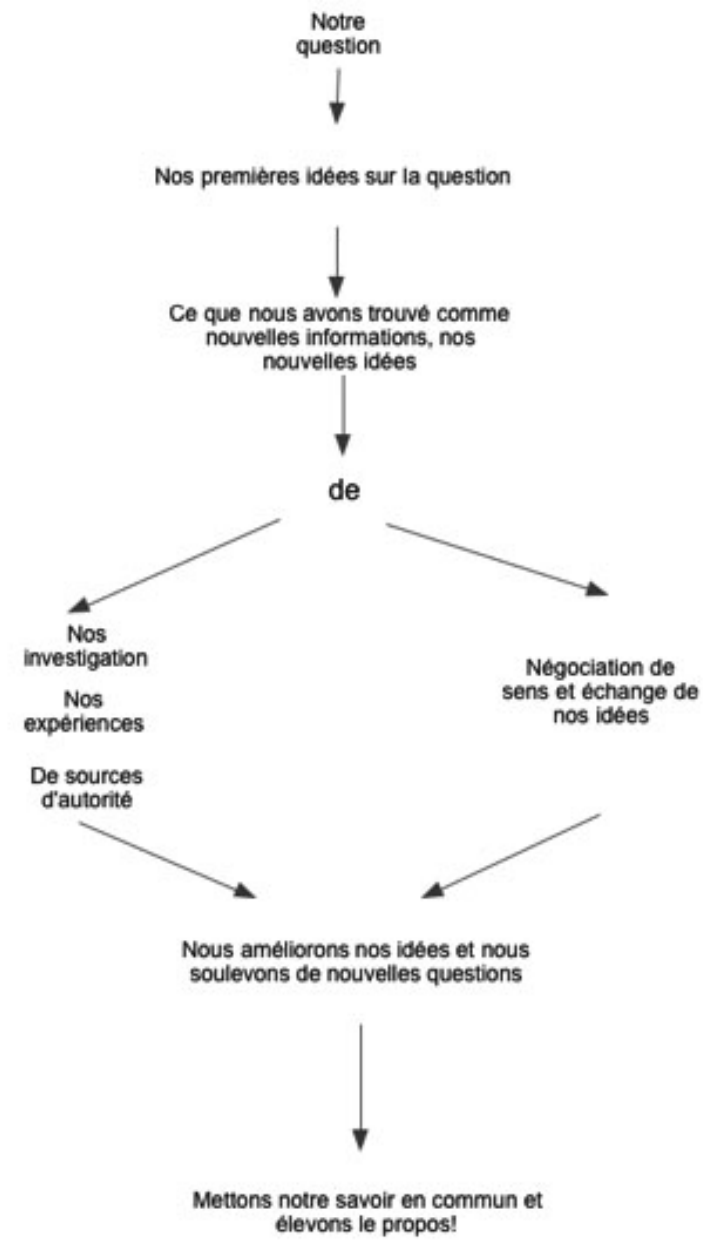

Figure 2 : Les actions de base posées dans un discours collectif (inspiré de Bielaczyc et Ow, 2013)

Ainsi, afin de mieux comprendre l'espace de réflexion offert aux futurs enseignants dans le contexte du cours, notre étude vise à documenter:

1. Quel est le niveau d'engagement des participants dans le discours collectif?

2. Quels sont les types d'interaction entre les participants ?

3. Quelles sont les actions de base posées par les participants dans la construction du discours collectif?

4. Quelles sont les phases de la construction de connaissances dans l'espace collectif partagé par les étudiants?

\section{Méthodologie}

\subsection{Devis de l'étude - Déroulement}


La présente étude de cas (Yin, 2015) vise à documenter le niveau d'engagement, les types d'interactions, les actions posées de même que les phases de coconstruction de connaissances dans le discours collectif d'une cohorte d'étudiants en enseignement au secondaire $(n=181)$ dans le cadre d'un cours de gestion de classe se déroulant sur deux semestres universitaires.

\subsection{Analyse de données}

Les contributions étudiantes analysées sont issues du travail effectué au retour d'un stage de cinq semaines. Nous avons analysé le discours collectif de 97 contributions centrées sur « la curiosité des élèves (ou le conflit cognitif ou la motivation des élèves à apprendre) », ce qui représente $15 \%$ des contributions disponibles dans l'environnement d'apprentissage.

Nous avons également utilisé les types d'interactions (explicites, implicites et indépendantes) des étudiants pour analyser les contributions au discours collectif. Les interactions explicites concernent les contributions qui font une référence explicite à une contribution d'un pair ou à son auteur. Les interactions implicites sont, quant à elles, considérées comme des contributions faisant une référence implicite aux contributions des pairs, par exemple par un lien d'élaboration (enfilade), mais sans préciser le nom de l'auteur ou de la contribution en question. Les interactions indépendantes sont des contributions sans lien d'élaboration et sans référence à un autre auteur ou à une autre contribution.

Les différentes actions (Tableau 1) ont été utilisées afin d'analyser le contenu des contributions faites au discours collectif par les étudiants. Ainsi, à l'intérieur d'une contribution plusieurs actions ont pu être posées. L'unité de sens de ces analyses était plus fine et concernait les idées plutôt que la contribution entière. Nous comprenons les idées comme un ensemble de mots, phrases, paragraphes poursuivant une même intention d'écriture, un même sens. Elles sont donc considérées comme des unités de sens propositionnelles, c'est-à-dire qui sont délimitées selon des relations sémantiques entre des concepts particuliers, ayant une forme propositionnelle particulière (Krippendorff, 2009). Nous avons analysé les contributions ( $n=97$ ) des étudiants en repérant les différentes actions posées telles que décrites dans le tableau 2.

\section{Tableau 1 : Actions posées par les étudiants}

\begin{tabular}{ll}
\hline Actions posées & Exemples \\
\hline Pose une question & $\begin{array}{l}\text { Comment faire pour que les activités en classe, l'évaluation et l'enseignant, eux- } \\
\text { mêmes, suscitent la motivation des élèves en difficulté d'apprentissage? }\end{array}$ \\
& $\begin{array}{l}\text { Ensuite, je souhaite intervenir sur « la logique d'imposer ce genre de cours } \\
\text { (mathématiques) aux élèves alors qu'ils n'ont même pas à le réussir. » J'ai }\end{array}$ \\
l'impression en lisant ce passage que l'enseignement se limite à la transmission de \\
connaissances. Ainsi, un élève n'ayant pas la motivation d'augmenter son bagage \\
de savoirs n'a aucun intérêt à fréquenter les institutions scolaires. Encore ici, j'ai
\end{tabular}

Réfère à la contribution d'un pair

Pour répondre à ta question, Gilles, je crois que l'enseignement de la poésie a une portée beaucoup plus large que le simple fait d'amener l'élève à identifier des alexandrins ou encore à utiliser des figures de style dans ses écrits. En fait, un parallèle peut être établi ici avec la contribution de Maude.

Réfère à une source d'autorité

Ceci va dans le même sens que les propos de Rolland Viau par rapport à la motivation en contexte scolaire. Selon lui, elle se construit avec « les perceptions que l'élève a de la valeur d'une activité, de sa compétence et de la contrôlabilité de cette activité ».

Fait une synthèse

Suite aux contributions de tout le monde, j'ai essayé de me faire un résumé, ou du moins un rassemblement, de nos savoirs, de nos expériences et de nos croyances à propos de la motivation de nos élèves.

*inspiré de Bielaczyc et Ow, 2013 
Pour entrer plus en profondeur dans les mécanismes du processus d'élaboration du discours collectif, nous nous sommes tournés vers les recherches de Wise et Chiu (2011) et avant eux Gunawardena, Lowe et Anderson (1997) sur le processus collectif de construction de connaissances. Nous avons analysé les contributions faites au discours collectif selon les «Phases de la construction de connaissances » telles que décrites dans le tableau 3. Certaines catégories ont dû être ajustées à notre contexte, par exemple dans la catégorie «Essais » les étudiants réfèrent souvent à des expériences passées (lors de leur stage III s'étant déroulé avant le travail collectif sur le KF) pour modifier ou appliquer les synthèses proposées dans le discours collectif.

\begin{tabular}{ll}
\hline $\begin{array}{l}\text { Phases de la construction de } \\
\text { connaissances }\end{array}$ & Description \\
\hline Partage d'informations & $\begin{array}{l}\text { Déclarations d'observation, d'opinion, d'accord, la clarification, par } \\
\text { exemple, ou la définition du problème, etc. }\end{array}$ \\
$\begin{array}{l}\text { Identification des zones de désaccord; la clarification de la source et } \\
\text { l'étendue du désaccord; fournir un soutien pour ses idées face au } \\
\text { contrargument. }\end{array}$ & $\begin{array}{l}\text { Zones d'identification de l'accord à travers des idées contradictoires; } \\
\text { clarification des significations des termes; proposition et de négociation de } \\
\text { l'intégration des métaphores et des déclarations de compromis. }\end{array}$ \\
$\begin{array}{l}\text { Négociation - coconstruction de } \\
\text { connaissances }\end{array}$ & $\begin{array}{l}\text { l'expérience personnelle, les données recueillies, et des témoignages } \\
\text { d'experts. }\end{array}$ \\
$\begin{array}{l}\text { Essais - modification des synthèses } \\
\text { proposées - }\end{array}$ & $\begin{array}{l}\text { Résumé de l'accord; application de nouvelles connaissances; déclarations } \\
\text { métacognitives de l'évolution des connaissances ou des façons de penser. }\end{array}$ \\
\hline $\begin{array}{l}\text { Accord - application des nouvelles } \\
\text { connaissances }\end{array}$ &
\end{tabular}

(Wise et Chiu, 2011 ; Gunawardena, Lowe et Anderson, 1997)

\section{Résultats et discussion}

\subsection{Le niveau d'engagement et les types d'interaction des étudiants dans le discours collectif}

Nous avons identifié les contributions liées à la participation (2\%). Il s'agit des contributions qui ne sont liées à aucune autre par un lien d'élaboration. Par exemple, un étudiant peut avoir posé une question ou une problématique qui n'a pas attiré l'attention ou l'intérêt des autres participants. Nous avons également relevé les contributions de l'ordre de l'interaction (10\%), qui ont un deuxième niveau d'arborescence. Ces contributions sont parfois de l'ordre de «la bonne réponse» ou du monologue (Henri, 1995) ou n'ont simplement pas fait l'objet d'une élaboration.

Les contributions de l'ordre de la collaboration sont les plus présentes et représentent $88 \%$ des contributions faites par les étudiants. Les contributions « collaboration » sont celles ayant atteint minimalement une arborescence de trois niveaux. Le niveau de discussion "Collaboration » étant le plus présent dans notre analyse du discours, nous pourrions être amenées à penser que les moyens mis en oeuvre pour engager les étudiants dans un discours signifiant concernant leur pratique professionnelle et leur réflexion quant à la gestion d'une classe au secondaire ont été féconds.

Les types d'interactions (Henri 1992, 1995) sont eux aussi liés au niveau d'arborescence (interactions implicites et indépendantes), mais s'attardent davantage au contenu des contributions. Ainsi, les interactions implicites, celles qui ont un lien avec une contribution d'un pair, mais sans en faire une mention explicite dans son contenu représentent $52 \%$ des interactions dans les 97 contributions. Les interactions explicites sont quant à elles présentes dans $46 \%$ des contributions. Rappelons que les interactions explicites concernent les contributions qui ont un lien explicite avec une autre contribution du discours dans leur contenu, tel que : "J'aimerais poursuivre sur une des pistes lancées par Sabrina qui nous apprend que, ici je la cite et cite le livre de nouveau [...] », et " Ce que dit Julie est tout à fait vrai. Pour compléter cette idée, j'ajouterais que peu importe les activités intéressantes qu'on pourra leur proposer, les élèves auront un rapport stratégique vis-à-vis de l'école ». Les interactions indépendantes sont présentes dans $4 \%$ des contributions. Ainsi peu de contributions sont déconnectées du discours collectif au sens où elles ne sont ni une élaboration, ni une contribution qui a amené une ou des élaborations, ce qui peut être un indice d'un discours collectif signifiant pour la communauté d'apprenants que formaient les étudiants. Nous supposons que les contributions faites au discours dans la perspective analysée étaient, pour la presque totalité, des contributions de valeur 
pour les participants cohésif, collectif audes élèves.

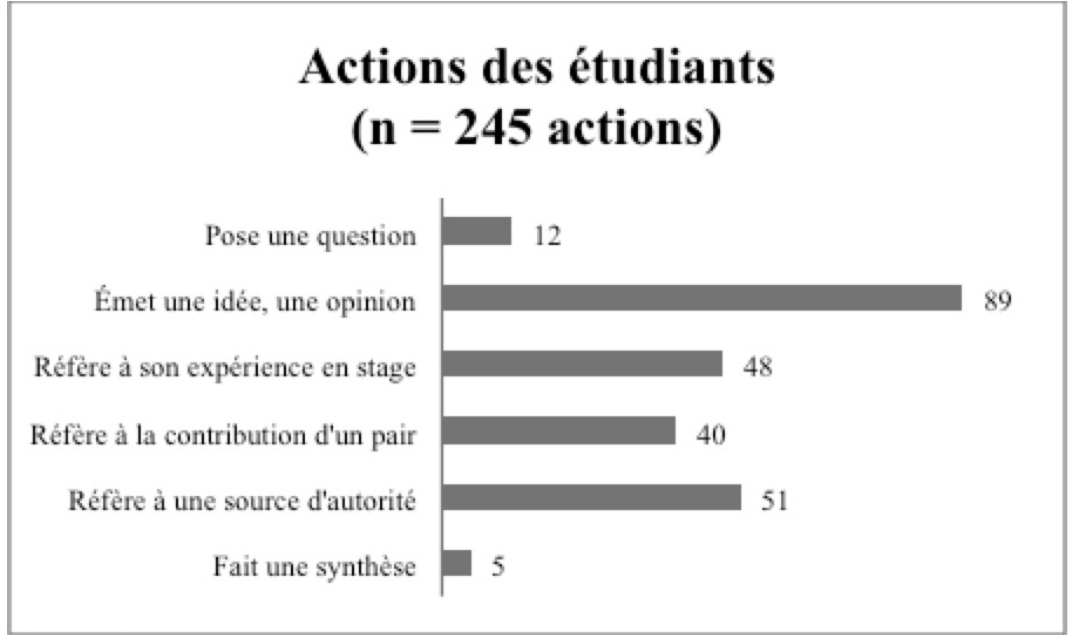

qui ont construit un discours lié, tour la curiosité et la motivation

Figure 3 : Les types d'interactions

\subsection{Les actions posées par les étudiants dans le discours collectif}

Pour faire suite aux interactions dans le discours, nous nous sommes penchées sur les actions posées par les étudiants dans leurs contributions au discours collectif. Pour les analyser, nous nous sommes inspiré des travaux de Bielaczyc et Ow (2014) tel qu'illustré par la figure 2. Nous avons donc relevé 245 actions dans les 97 contributions soumises.

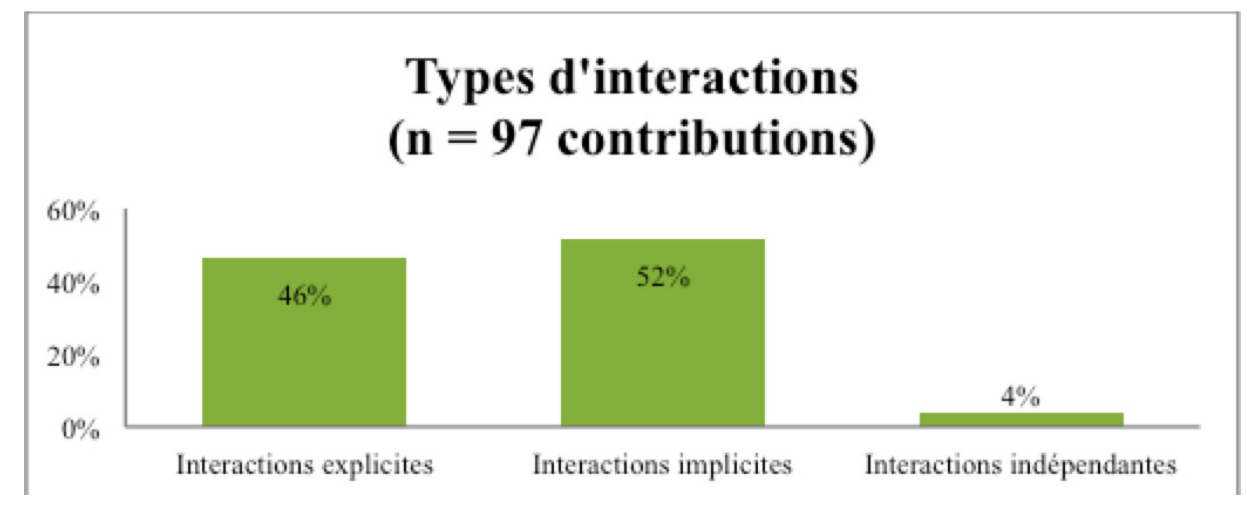

Figure 4 : Les actions posées par les étudiants lors de l'élaboration du discours collectif

Les étudiants émettent une idée ou une opinion dans $92 \%$ d'entre elles, soit un total de 89 contributions. Dans le cas qui nous occupe, il y a rien d'étonnant puisque les idées sont au cœur du processus du discours collectif et sont la pierre angulaire de la coélaboration de connaissances. Nous entendons par idée ou opinion un ensemble de mots ne faisant explicitement référence à aucune source d'autorité, pairs ou expérience, c'est l'expression des valeurs, intuitions, réflexions des étudiants. Voici un exemple d'idées de deux étudiants au sujet de la curiosité et de la motivation :

- J'ai l'impression en lisant ce passage que l'enseignement se limite à la transmission de connaissances. Ainsi, un élève n'ayant pas la motivation d'augmenter son bagage de savoirs n'a aucun intérêt à fréquenter les institutions scolaires. Encore ici, j'ai l'impression de faire un long retour 30 ans en arrière.

- Je me demande donc s'il serait intéressant de passer à travers le programme de mathématiques du secon- 
daire avec eux. Par la suite, ils pourraient identifier avec quelle portion de matière ils ont eu de la facilité. Ainsi, je pourrais voir avec eux quelles sont leurs forces et tenter de les retrouver dans chaque portion de matière que nous voyons. Je trouve que cela serait une activité intéressante à faire en début d'année avec les groupes en difficultés. De plus, cela nous aiderait aussi à connaitre nos élèves.

Les idées et opinions ainsi émises dans le discours s'accompagnent généralement de références à des sources d'autorités et à la contribution d'un pair et/ou à une expérience de stage (près de $91 \%$ des contributions contenant des idées s'accompagnent d'une ou plusieurs références à des sources d'autorité, aux propos des pairs ou à une expérience vécue en stage). Ainsi, 51 \% des contributions contiennent une référence à une source d'autorité, par exemple : « Avant de faire le lien avec cette situation vécue, je vous propose une définition de Weigner [...] ». "La motivation de l'élève pour les activités scolaires peut être qualifiée, selon la classification instaurée par Deci (1975), d'intrinsèque si ces activités sont accompagnées d'un sentiment de compétence et d'autodétermination, et qu'elles procurent en elles-mêmes intérêt et satisfaction » et :

Dans la même veine, Thompson et Hamel (2012, pp. 221,222) dressent une liste d'éléments susceptibles de susciter la curiosité des élèves. De plus, la notion de signifiance vient aussi jouer un rôle important lorsque survient un commentaire du type : "C'est inutile! » Effectivement, selon Samson (2002), l'enseignant doit présenter " [...] des situations près des élèves afin de soutenir la motivation scolaire, de créer un besoin d'apprendre [...]»

Près d'une contribution sur deux (48\%) relate une expérience de stage pour bonifier une explication, appuyer une idée ou pour la remettre en question, en voici un exemple :

- Durant mon stage, j'ai aussi eu à faire face à des élèves très peu motivés, surtout dans un groupe de deuxième secondaire où sensiblement la moitié des élèves étaient des doubleurs. Ils ne participaient pas aux discussions, ne prenaient pas de notes et ne se donnaient même pas la peine de lire la consigne pour faire les activités.

- J'ai travaillé longuement avec une élève qui éprouvait de grandes lacunes en compréhension de lecture et en interprétation. Je lui ai présenté les stratégies de lecture dans plusieurs textes, l'élève a fait trois examens formatifs et un examen sommatif. Au début du processus, elle était peu motivée, puisqu'elle ne comprenait pas l'intérêt d'utiliser ces stratégies lors de la lecture d'un texte. Toutefois, à la suite du modelage que j'ai fait avec elle, elle a compris que celles-ci permettaient de mieux le comprendre et d'être en mesure de l'analyser.

$41 \%$ des contributions contiennent une référence aux propos d'un pair. Les étudiants y font référence pour les bonifier, manifester leur accord ou pour les remettre en question. Par exemple :

- Ce que dit Julie est tout à fait vrai. Pour compléter cette idée, j'ajouterais que peu importe les activités intéressantes qu'on pourra leur proposer, les élèves auront un rapport stratégique vis-à-vis de l'école.

- Dans sa contribution du 15 mars, Annie apporte un exemple d'une séquence d'enseignement qu'elle a mise en place dans l'un de ses cours durant son stage III. J'y vois une source inspirante de levier pour augmenter la motivation des élèves en sortant du contexte brut de la progression des apprentissages.

- Je voudrais faire un retour par rapport à ce que tu as dit dans ton premier paragraphe et je cite: «II serait facile d'accuser ces adolescents qui ne font rien, mais, en grattant un peu, j'ai trouvé que les responsables de tout ça sont les enseignants ».Je trouve que tu tires une conclusion plutôt hâtive... Pourquoi ce ne serait pas l'inverse qui est vrai? Pourquoi les enseignants n'auraient-ils pas perdu le goût d'enseigner parce que les nouvelles générations sont paresseuses?

Un peu plus d'une question sur dix (12\%) est en lien avec les idées d'un pair, ou visant à relancer la discussion : "Comment mettre cette théorie en pratique? Avez-vous tenté des expériences en stage? » «Que faire lorsqu'ils se disent incapables de ne rien faire et ne font pas le moindre petit effort pour essayer de réussir? Avez-vous eu à travailler avec des groupes plus difficiles qui ne voulaient pas collaborer? Qu'avez-vous fait? » Peu de contributions contiennent des synthèses (5\%), elles proposent le plus souvent un résumé des propos tenus plus tôt dans le discours pour introduire de nouveaux faits, idées, explications :

- Plusieurs ont accusé les élèves d'être la principale cause du problème, d'être paresseux, démotivés et de ne faire des efforts que lorsqu'il y a une récompense au bout. D'autres ont mentionné les enseignants comme principale cause du problème, les accusant de rester ancrés dans une routine et de ne pas faire d'efforts pour se renouveler et intéresser les élèves. Certains ont même mentionné que le problème provenait de la société telle qu'elle est aujourd'hui : une société de consommation où tout doit aller vite et où 
tout est jetable et remplaçable

- Suite aux contributions de tout le monde, j'ai essayé de me faire un résumé, ou du moins un rassemblement, de nos savoirs, de nos expériences et de nos croyances à propos de la motivation de nos élèves. J'ai trouvé un article de Rolland Viau (1998) qui donne diverses catégories de facteurs qui influencent la motivation des élèves. J'ai l'impression que cette nomenclature regroupe bien les divers aspects abordés dans la discussion en lien avec la motivation.

II nous semble tout à fait réaliste que le nombre de synthèses soit moins élevé que le nombre d'idées ou de références dans un discours collectif. En effet, les synthèses se composent autour d'idées qui sont plus nombreuses et qui peuvent être regroupées. Cependant, aucune grande synthèse des idées présentes, des différents points de vue sur la curiosité et la motivation, ne semble avoir été proposée par les étudiants. Les synthèses parsèment le discours et font le résumé d'enfilades ou de groupe de contributions sans porter un regard global sur le discours.

\subsection{Les phases de construction de connaissances dans le discours collectif}

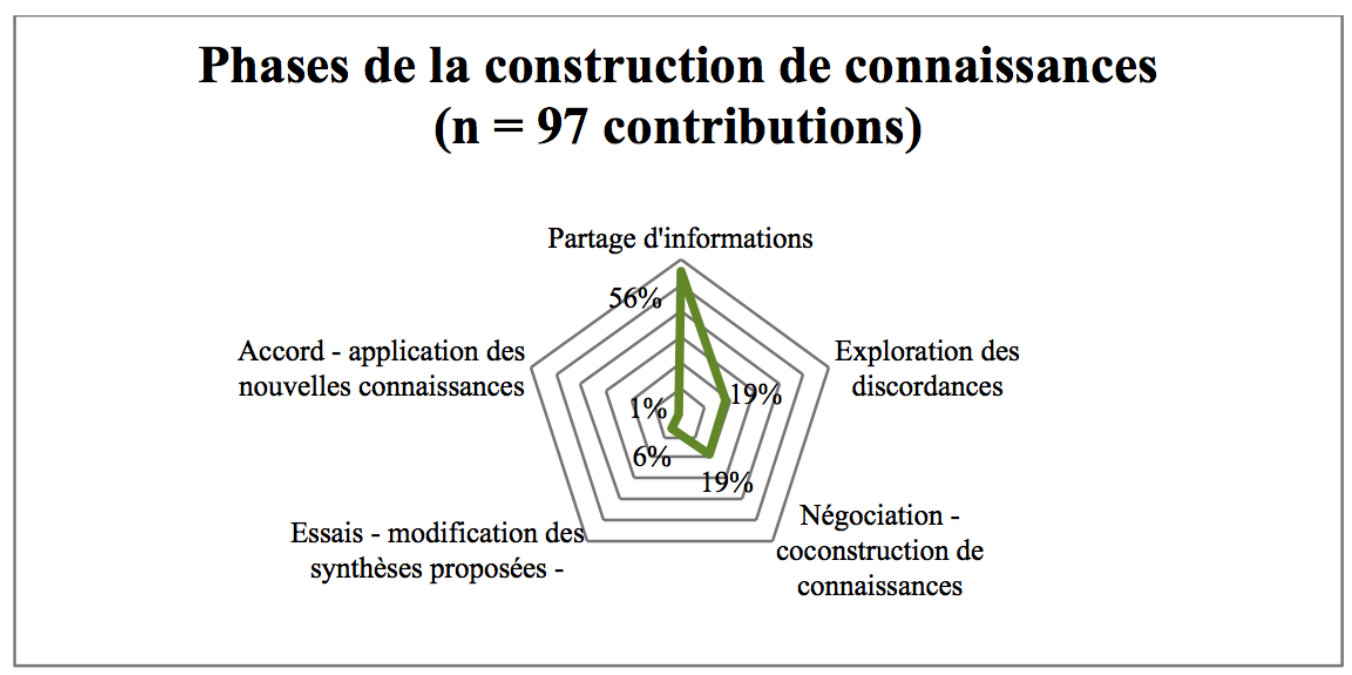

Figure 5 : Les phases de la construction de connaissances présentes dans le discours collectif

Nous avons observé qu'un peu plus d'une contribution sur deux (56 \%) faisait du partage d'informations, c'est-à-dire qu'elles apportaient de nouvelles informations, idées, opinions. Ce qui est tout à fait cohérent avec le nombre de contributions, 89 sur un total de 97 émettant des idées. Cependant, on remarque que la proportion des contributions de la phase "Partage d'information » est moins élevée que celle des contributions contenant des idées et des opinions. En effet, les idées et les opinions sont présentes dans toutes les phases de la construction de connaissances, elles sont au cœur du processus de négociation de sens et ce sont elles qui font avancer le discours. Dans $19 \%$ des contributions, les étudiants soulèvent et explorent les discordances dans leur discours. Par exemple, ici l'étudiante remet en question le rôle de l'enseignant dans la motivation des élèves, elle se questionne:

- Oui, le dynamisme de l'enseignant peut être contagieux et faciliter le travail en classe, mais pour toucher à la curiosité intellectuelle, il faut s'assurer que l'élève puisse associer un sens au développement de ses compétences. Apprendre pour le plaisir d'apprendre, il faut l'avouer, n'est pas la tasse de thé de tous, et encore moins pour les élèves de secondaire 3.

Il est intéressant de constater que bien que les étudiants ne se trouvaient pas dans un discours de débat, certains ont néanmoins soulevé des désaccords. Cette phase d'exploration des discordances les amène ensuite à négocier le sens de certains concepts relatifs à la curiosité et à la motivation des élèves et leurs liens possibles avec la gestion de classe. Cette phase leur permet donc de prendre position, mais aussi de remettre en question leurs idées et celles des autres dans le but de faire avancer l'état de leurs connaissances. Dans près d'une contribution sur cinq, soit $19 \%$, la communauté élabore ses connaissances par la négociation de sens. Dans l'exemple suivant, l'étudiant, en plus de mettre en relief les différents points de vue, va plus loin et négocie le sens du concept de motivation chez les élèves en proposant une comparaison:

- Plusieurs ont accusé les élèves d'être la principale cause du problème, d'être paresseux, démotivés et de ne 
faire des efforts que lorsqu'il y a une récompense au bout. D'autres ont mentionné les enseignants comme principale cause du problème, les accusant de rester ancrés dans une routine et de ne pas faire d'efforts pour se renouveler et intéresser les élèves. Certains ont même mentionné que le problème provenait de la société telle qu'elle est aujourd'hui : une société de consommation où tout doit aller vite et où tout est jetable et remplaçable. Et selon moi, tous ont un peu raison, car aucune de ces causes n'explique en elle-même le problème de motivation perçu dans les écoles. Les élèves ne veulent pas travailler sans en voir l'utilité? Qui pourrait leur en vouloir! Moi non plus je n'aime pas travailler sans savoir pourquoi ou encore de mettre des efforts dans le vide. J'aime travailler pour atteindre un but, j'aime voir des résultats et avoir un sentiment d'accomplissement suite à mes efforts. Il en va de même pour les élèves et c'est normal.

Peu de contributions (6\%) faisaient état d'une modification des synthèses proposées. Ceci peut s'expliquer par le fait que les étudiants revenaient d'un stage de cinq semaines et ne se trouvaient plus, lors de la participation au forum, dans un contexte de pratique où ils auraient pu réinvestir la nouvelle connaissance négociée. Néanmoins, certains se sont revenus sur leur expérience, ou celle de leurs pairs, de stage afin de vérifier les synthèses proposées dans le discours collectif. Ici, plusieurs en arrivaient à la conclusion qu'il faut être passionnés pour motiver les élèves, l'étudiant remet en question le concept négocié et avance de nouvelles pistes en évoquant son expérience en stage :

- Malheureusement, comme tu le mentionnes au début de ta note, nous devons parfois enseigner des notions pour lesquelles nous avons beaucoup moins d'intérêts, et c'est ce qui m'est arrivé en stage. Mon enseignante associée m'avait d'ailleurs avertie que le concept en question (les types de sols) n'était jamais vraiment apprécié par les élèves, et elle accusait justement son manque d'intérêt sur le sujet. N'étant moi-même pas très fanatique de géologie, j'aurais pu rencontrer le même problème si je n'avais pas eu la chance de pouvoir utiliser une SAÉ stimulante que j'avais conçue dans le cadre de mon cours de didactique des sciences. Cette SAÉ permettait aux élèves de réaliser des apprentissages sur les types de sol sans mon intervention directe. En gros, les élèves avaient à jouer le rôle d'un pomiculteur. [...] Je pense que de contextualiser les apprentissages comme je l'ai fait, même s'il s'agissait d'un contexte fictif, a contribué à donner du sens à leurs apprentissages et à stimuler leur intérêt.

Pour ce qui est des contributions faisant «I'application » (phase 5) de nouvelles connaissances, une seule (1\%) a été dénombrée dans le discours collectif. II va sans dire qu'il est ardu d'en arriver à une phase d'accord explicite lors de l'élaboration d'un discours écrit auquel participe un très grand nombre d'étudiants. Ainsi, la phase d'application est représentée par la première contribution faite dans l'espace de travail sur la curiosité et la motivation des élèves. En effet, cette contribution, rédigée par la professeure responsable du cours de gestion de classe, était de nature métacognitive au sens où elle soulevait le chemin parcouru par les participants et les amenait sur le consensus obtenu en ce qui concerne la curiosité et la motivation. Les contributions au discours collectif faisant partie des phases plus avancées, négociation, modification et application, sont moins nombreuses dans le discours. En effet, la néqociation, la modification et l'applicatic

Tablec

Pose une Emet une Réfère à son

Réfère à la Réfère à une

idée, une expérience en

contribution

source

Fait une er plus loin.

question

stage

d'un pair

d'autorité

synthèse snnaissances

\section{Partage \\ d'informations \\ Exploration des \\ discordances \\ Négociation - coconstruction de connaissances \\ Modification - essai des synthèses proposées}

Application Consensus autour des nouvelles connaissances
$2,9 \%$

$20,0 \%$

$7,8 \%$

$0,4 \%$

$0,8 \%$

$6,1 \%$

$2,9 \%$

$4,9 \%$

$2,9 \%$

$1,2 \%$

$0,4 \%$

$2,0 \%$

$1,2 \%$

$1,6 \%$

$1,6 \%$

$0,0 \%$

$0,4 \%$

$0,4 \%$

$0,4 \%$

$0,4 \%$

$0,0 \%$

$0,4 \%$ 
Comme la plupart des contributions (56\%) ont été réalisées dans la phase « Partage d'informations », il n'est pas étonnant d'y retrouver un fort pourcentage des idées et opinions émises par les étudiants (20\%), les idées sont également présentes, dans une proportion moins grande, mais non négligeable dans les phases d'exploration (7,8\%), de négociation (6,1\%), de modification (2\%) et d'application (0,4\%). Les références sont également bien représentées dans la phase de partage d'information avec $12,7 \%$ pour les références aux sources d'autorités, les références à une expérience de stage représentent 12,2\%. Ce sont les références aux contributions des pairs qui y sont le moins représenté avec 5,3\%. Les questions sont aussi présentes dans la phase de partage en pourcentage plus élevé que dans les autres phases de construction de connaissances avec 2,9\%. Les références à la contribution d'un pair sont plus présentes que les autres types de références dans les phases de négociation $(4,9 \%)$, pour faire référence aux propos d'un pair autour duquel on négocie le sens d'un ou plusieurs concepts relatifs à la gestion de classe, d'exploration (4,1\%), pour référer à la contribution d'un pair afin d'y soulever les points avec lesquels l'étudiant n'est pas d'accord, de modification (1,6\%) et d'application (0,4\%). Les synthèses sont les actions les moins représentées dans le discours (1\%). Les autres actions posées par les étudiants apparaissent dans des proportions négligeables $(0,4 \%$ à $1,6 \%)$ dans les phases de modification et d'application, par contre, on dénote une légère augmentation pour ce qui est des synthèses qui apparaissent surtout durant la phase de négociation (1,2\%). Ce qui signifie que les étudiants ont fait des synthèses lors de la négociation de sens plutôt que dans les phases de modification et d'application. Ceci peut être dû au fait que les contributions des deux dernières phases sont très peu nombreuses (7 \% des contributions).

Le peu de synthèse présente dans le discours des étudiants, de même qu'une certaine concentration dans des actions centrées sur l'opinion et les références à des experts démontrent qu'un étayage doit être fourni de manière constante dans ce type d'espace professionnel. Ainsi, même si les étudiants démontrent une capacité à construire des connaissances, que restera-t-il de ces interactions au niveau individuel ? Au-delà des exigences du cours, entre autres le fait de faire des liens avec les théories, est-ce que cet espace professionnel peut conduire à une appropriation d'un discours professionnel soutenu ? Nous croyons qu'il s'agit d'un point de départ pour permettre aux futurs enseignants de négocier le sens de leur pratique entre pairs, dans un métier où cela n'est pas toujours possible, notamment lors de l'insertion professionnelle. Toutefois, nous demeurons consciente que cet espace en construction vit de manière intensive parce qu'il fait partie des exigences formelles du cours. II demeure qu'il s'agit du seul espace écrit dans lequel les futurs enseignants, du moins dans notre institution, sont soumis à des exigences en matière de collaboration professionnelle et d'approfondissement de concepts. Rendre visible ses idées dans un discours collaboratif auprès d'un collectif de travail nous semble prometteurs pour soutenir leur professionnalisation.

\section{Conclusion}

Le fait d'interagir pour apprendre le métier d'enseignant, plus particulièrement la gestion de classe au secondaire, nous semble prometteur dans le contexte de notre design pédagogique. Ainsi, bien que les contributions au discours collectif faisant partie des phases plus avancées, comme la négociation, la modification et l'application, sont moins nombreuses dans le discours, il demeure qu'il s'agit de processus très avancés qui ne peuvent probablement pas se réaliser en un aussi court laps de temps. En effet, la négociation, la modification et l'application s'ancrent à certaines idées et désaccords présents dans le discours collectif pour les approfondir, et il est souvent plus difficile lors d'une première immersion dans un tel design d'y parvenir. Les phases les plus avancées de la construction de connaissances (modification et application) sont limitées par le contexte de notre étude puisque les étudiants ne retournent pas dans la pratique (en stage) pour mettre en application « leurs discussions ». II faudrait aussi s'intéresser au lien entre le discours construit par les étudiants et le lien entre les compétences professionnelles à développer durant la formation.

Le partage d'un espace collectif, et l'analyse du discours d'une portion de celui-ci, nous semble tout de même être un design prometteur pour soutenir des interactions porteuses de sens puisque nos données démontrent clairement que les étudiants se sont engagés, sans être des participants périphériques dans le discours. La mise en place de ce type de design donnerait probablement plus de résultats s'il était plus présent dans la formation initiale des enseignants. Malgré cette limite temporelle et expérientielle, les résultats tendent à démontrer que les étudiants non seulement participent et interagissent, mais collaborent également entre eux pour approfondir leur compréhension des enjeux liés à la gestion de classe. Bien entendu, il s'agit d'un contexte formel de formation dans lequel la participation au forum fait partie des exigences du cours. II demeure que les étudiants se sont appuyés sur des auteurs, mais également sur leurs pratiques et sur la pratique de leurs pairs pour étayer et négocier leurs conceptions. Ainsi, nous croyons qu'un espace collectif d'interactions professionnelles peut être porteur de 
sens dans la professionnalisation des futurs enseignants. Pour la suite, il serait pertinent d'analyser le discours collectif des étudiants au retour de leur dernier stage en enseignement, le stage IV qui se déroule sur quinze semaines, quant à la transposition de ce discours collectif dans leurs pratiques réelles.

\section{Références bibliographiques}

Alexander, G. (1992). Designing human interfaces for collaborative learning. Dans A.R. Kaye (dir), Collaborative learning through computer conferencing: the Najaden papers (p. 201-210). New York, NY : Springer.

Allaire, S., Thériault, P., Gagnon, V., Laferrière, T., Hamel, C., Boutin, P. \& Debeurme, G. (2014). Vers une écriture collective transformative au primaire : interventions enseignantes et design technologique. Science et technologies de l'information et de la communication pour l'éducation et la formation, 20, 1-24.

Bereiter, C. \& Scardamalia, M. (1987). The psychology of written composition. Hillsdale, N-J: Lawrence Erlbaum Associates.

Bereiter, C. \& Scardamalia, M. (2010). Can Children Really Create Knowledge? Canadian Journal of Learning and Technology, $36(1), 1-24$.

Bielaczyc, K \& Ow, J. (2014). Multi-player epistemic games: Guiding the enactment of classroom knowledge-building communities. International Journal of Computer-Supported Collaborative Learning, 9(1), 33-62.

Bransford, J. D., Brown, A. L. \& Cocking, R. R. (2000). How people learn: Brain, mind, experience, and school. Washington, DC : National Academies Press.

Brown, A. L. (1994). The Advancement of Learning. Educational Researcher, 23(8), 4. doi:10.2307/1176856

Cazden, C. B. (2001). Classroom discourse: The Language of Teaching and Learning. Portsmouth, NH : Heinemann.

Gunawardena, C., Lowe, C. A. \& Anderson, T. (1997). Analysis of Global Online Debate and the Developpement of an Interaction Analysis Model for Examine Social Construction of Knowledge in Computer Conferencing. Journal of Educational Computing Research, 17(4), 397-431.

Henri, F. (1992). Computer Conferencing and Content Analysis. Dans A.R. Kaye (dir), Collaborative learning through computer conferencing: the Najaden papers (p. 117-136). New York, NY: Springer.

Henri, F. (1995). Distance Learning and Computer-Mediated Communication: Interactive, Quasi-Interactive or Monologue? Dans C. O'Malley (dir), Computer Supported Collaborative Learning (p. 145-161). Berlin, D : Springer-Verlag.

Korthagen, F., Loughram, J. \& Russel, T. (2006). Developing fundamental principles for teacher education programs and practices. Teaching and Teacher Education, 1020-1041.

Laferrière, T. \& Lamon, M. (2010). Knowledge Building/Knowledge Forum : The Transformation of Classroom Discourse. Dans M. S. Khine \& I. M. Saleh (dir), New Science of Learning. New York, NY: Springer..

Mugny, G. \& Doise, W. (1978). Socio-cognitive conflict and structure of individual and collective performances. European Journal of Social Psychology, 8(2), 181-192.

Murphy, E. (2004). Recognising and promoting collaboration in an online asynchronous discussion. British Journal of Educational Technology, 35(4).

Paavola, S., Lipponen, L. \& Hakkarainen, K. (2004). Models of Innovative Knowledge Communities and Three Metaphors of Learning. Review of Educational Research, 74(4), 557-576.

Perkins, D. N. (1995). L'individu-plus: Une vision distribuée de la pensée et de l'apprentissage. Revue Française de Pédagogie, 111(1), 57-71.

Perrenoud, P. (2001). Mettre la pratique réflexive au centre du projet de formation. Cahiers Pédagogiques, (390), 42-45.

Scardamalia, M. (2004). CSILE/Knowledge forum $®$. Dans Education and technology: An encyclopedia (p. 183-192). Santa Barbara, CA : ABC-CLIO.

Scardamalia, M. \& Bereiter, C. (2003). Knowledge building: Theory, pedagogy, and technology. Dans Encyclopedia of Education (p. 1370-1373). New York, NY : Macmillan.

Sfard, A. (1998). On two metaphors for learning and the dangers of choosing just one. Educational Researcher, $27(2), 4$.

Schön, D. (1983). The reflective practitioner : How professionals think in action. New York, NY: Basic Books.

Tardif, J. (2012). Devenir ostéopathe. Agir avec compétence. St-Étienne, France : SNESO.

Tardif, M. \& Desbiens, J.-F. (dir). (2014). La vogue des compétences dans la formation des enseignants. Sainte-Foy, QC : Presses de l'Université Laval.

Vygotsky, L. (1978). Mind and society. Cambridge: MA : Harvard University Press.

Wise, A. F. \& Chiu, M. M. (2011). Analyzing temporal patterns of knowledge construction in a role-based online discussion. International Journal of Computer-Supported Collaborative Learning, 6(3), 445-470.

Wittorski. (2001). L'analyse des pratiques professionnelles : usages et formes. Conférence présentée le 24 mars 2011 dans le cadre d'une journée d'étude autour de l'axe 1 du CRIE, Université de Sherbrooke. Wood, D., Bruner, J. S. \& Ross, G. 